\title{
Integrated Design of an Active Torque Balancing Mechanism and a Planetary Gear Reducer*
}

\author{
Jing SUN** and Yanan YAO** \\ ** School of Mechanical and Electronic Control Engineering, \\ Beijing Jiaotong University, Beijing 100044, China \\ E-mail: yayao@center.njtu.edu.cn
}

\begin{abstract}
In this paper, a novel concept of integrating an active torque balancing mechanism with a planetary gear reducer is presented. This integrated device is composed of a speed reduction unit and a torque compensation unit. The speed reduction unit, which contains a two-stage planetary gear train, can make the device to transform the speed and torque for meeting the needed requirements of the machine. The torque compensation unit, which consists of a differential gear train and a servo motor, can make the device to balance the input torque fluctuations of the mechanical system. Through an analytical method, an exact control function which can totally eliminate the input torque fluctuation of the driving motor of the machine is derived for the servo motor of the integrated device. At the same time, by adjusting the structure parameters of the differential gear train, the torque fluctuation of the servo motor can be limited too. Besides, in order to obtain a satisfactory tradeoff between the torque fluctuations of the driving motor and the servo motor, an optimization method is developed to find an appropriate control function for the servo motor. In addition, an integrated approach is proposed to optimize both the structure parameters of the differential gear train and the control function of the servo motor. Two numerical examples are given to illustrate the design procedures and to show their feasibilities.
\end{abstract}

Key words: Torque Balance, Planetary Gear Reducer, Differential Gear Train, Integrated Design, Optimization Design

\section{Introduction}

It is well known that the gear reducer is a common device of the present day machinery, and is widely used in transforming the rotational speed and driving torque to meet needed requirements. Usually, this driving speed is desired to be constant with respect to time for the sake of reducing wear, decreasing vibration and noise levels, extending fatigue life and making the mechanism work precisely. However, since many working mechanisms have non-constant work requirements, when the torques accelerate and decelerate them, the driving speeds often exhibit substantial fluctuations.

Input torque balancing has been shown to be an effective method in dealing with the problem of driving speed fluctuations, and there is a large body of literature on this subject. Internal mass redistribution of the moving links and adding counterweights to the mechanisms were classified as "passive balancing" methods by Kochev ${ }^{(1)}$. They were applied to minimize the input torque fluctuation by optimizing inertial parameters of the mechanism. The "active balancing" methods ${ }^{(1)}$ were adding balancing devices like links ${ }^{(2)}$,

${ }^{*}$ Received 12 Feb., 2010 (No. 10-0074) [DOI: 10.1299/jsdd.4.391]

Copyright $\odot 2010$ by JSME 
cams $^{(3)-(7)}$, cams and springs ${ }^{(8)-(11)}$, non-circular gears ${ }^{(12)(13)}$ or more complex devices ${ }^{(14)(15)}$ to the working mechanism. Since most of the additional devices mentioned above are designed for different mechanisms, when the mechanism is changed by another one, the balancing device may not suit for the new mechanism. Furthermore, owning to the unavoidable errors of manufacturing and assembling, the mechanism in practice may not coincide with the designed one, and the corresponding balancing device may not reach the theoretical balancing effect. Moreover, if the working condition of the mechanism is altered, the balancing device may not be adapted to it and another device should be made for the new working condition.

During the recent years, there has been a growing interest in more active balancing methods. Kochev ${ }^{(1)}$ used a balancing disk which revolved in a prescribed way to balance the input torque. Thuemmel ${ }^{(16)}$ and Thuemmel and Brandl ${ }^{(17)}$ carried out the dynamic balance of joint forces by actively controlling the redundant drivers. Dyer and $\mathrm{Ni}^{(18)}$ proposed an adaptive control strategy for single-plane active balancing system of the rotating machinery to control steady-state rotation-synchronous vibration. In our previous work, some investigations were also implemented. A servo motor was applied to achieve the torque balancing of the linkage mechanism through varying the speed function of the input member actively ${ }^{(19)}$. A servo-controlled five-bar linkage was installed on the output shaft of the mechanism to improve the dynamic performance of the machine ${ }^{(20)}$. With considering the compact structure and the good dynamic performance of the gears, a differential gear train which is driven by a servo motor was employed and assembled on the output shaft of the working mechanism to reduce the torque fluctuation of the machine ${ }^{(21)}$.

The objective of this paper is to propose an innovative concept by integrating a balancing device which consists of a differential gear train and a servo motor within a gear reducer to be a more compact structural assembly. The new configuration can not only transmit the driving speed and torque to the working mechanism, but also minimize the torque fluctuation of the machine actively. When compensating the input torque fluctuation, the proposed integrated device will not disturb the desired kinematics characteristics of the mechanisms. Since the gear reducer is an indispensable application in machines, this new concept is expected to be widely used in the mechanical systems. Therefore, it may promote the industrial application of the balancing device.

\section{Nomenclature}

$\begin{array}{ll}\varphi_{d}, \varphi_{a} & : \text { angular displacements of gears } \mathbf{d} \text { and } \mathbf{a} \\ \dot{\varphi}_{d}, \dot{\varphi}_{a}, \dot{\varphi}_{b}, \dot{\varphi}_{H}, \dot{\varphi}_{g} & : \text { angular velocities of parts } \mathbf{d}, \mathbf{a}, \mathbf{b}, \mathbf{H} \text { and } \mathbf{g} \\ \ddot{\varphi}_{d}, \ddot{\varphi}_{a} & : \text { angular accelerations of gears } \mathbf{d} \text { and } \mathbf{a} \\ \varphi_{1} & : \text { angular displacement of the working mechanism's input member } \\ \dot{\varphi}_{1} & : \text { angular velocity of the working mechanism's input member } \\ i_{1} & : \text { transmission ratio of } \mathbf{d}-\mathbf{c} \\ i_{2} & : \text { transmission ratio of the two-stage planetary gear train } \\ i & : \text { transmission ratio of the whole integrated device } \\ E_{1} & : \text { kinetic energy of the working mechanism } \\ E_{2} & : \text { kinetic energy of the integrated device without the torque } \\ & \text { compensation unit } \\ E_{3} & : \text { kinetic energy of the differential gear train } \\ E^{\prime} & : \text { kinetic energy of the system without the torque compensation unit } \\ E & : \text { kinetic energy of the system with the torque compensation unit } \\ J_{e} & : \text { equivalent moment of inertia of the working mechanism } \\ J & : \text { equivalent moment of inertia of the device without the torque } \\ & \text { compensation unit }\end{array}$




\begin{tabular}{|c|c|}
\hline$M_{d}^{\prime}$ & $\begin{array}{l}\text { input torque of the driving motor in the system without the torque } \\
\text { compensation unit }\end{array}$ \\
\hline$J_{a}, J_{b}, J_{H}, J_{g}$ & : moments of inertia with respect to the centers of mass of $\mathbf{a}, \mathbf{b}, \mathbf{H}, \mathbf{g}$ \\
\hline$J_{g g}$ & $\begin{array}{l}\text { : moment of inertia of the planetary gear } \mathbf{g} \text { with respect to the center of } \\
\text { the sun gear } \mathbf{a}\end{array}$ \\
\hline$n$ & : the number of the planetary gears in the differential gear train \\
\hline$J_{11}, J_{12}, J_{22}$ & $\begin{array}{l}\text { : equivalent moments of inertia of the system with the torque } \\
\text { compensation unit }\end{array}$ \\
\hline$z_{a}, z_{b}, z_{c}, z_{d}, z_{g}$ & : teeth numbers of gears $\mathbf{a}, \mathbf{b}, \mathbf{c}, \mathbf{d}$ and $\mathbf{g}$ \\
\hline$M_{d}$ & $\begin{array}{l}\text { : input torque of the driving motor in the system with the torque } \\
\text { compensation unit }\end{array}$ \\
\hline$M_{a}$ & $\begin{array}{l}\text { : input torque of the servo motor in the system with the torque } \\
\text { compensation unit }\end{array}$ \\
\hline$R_{g}$ & : radius of the pitch circle of the gear $\mathbf{g}$ \\
\hline$r_{g}$ & : radius of the hollow circle of the gear $\mathbf{g}$ \\
\hline$m_{g}$ & : mass of the gear $\mathbf{g}$ \\
\hline$A$ & : center distance between the gears $\mathbf{a}$ and $\mathbf{g}$ \\
\hline$M$ & : module of the gears $\mathbf{a}, \mathbf{b}$ and $\mathbf{g}$ \\
\hline$\alpha$ & : pressure angle of the gears $\mathbf{a}, \mathbf{b}$ and $\mathbf{g}$ \\
\hline$h_{a}^{*}$ & : addendum coefficient of the gears $\mathbf{a}, \mathbf{b}$ and $\mathbf{g}$ \\
\hline . & : total time of a complete motion cycle \\
\hline
\end{tabular}

\section{Configuration}

The internal structure of the proposed integrated device is shown in Fig.1. It consists of the torque compensation unit and the speed reduction unit. The torque compensation unit contains a differential gear train and a servo motor. Since the planetary gear train is widely used in gear reducers and its structure is similar to the differential gear train, a two-stage planetary gear train is employed here as the speed reduction unit. The shaft $\mathbf{O}_{\mathbf{d}}$, which is the input shaft of the integrated device, is driven by the driving motor of the machine. The shaft $\mathbf{O}_{1}$, which is the output shaft of the integrated device, is connected with the input member of the working mechanism.

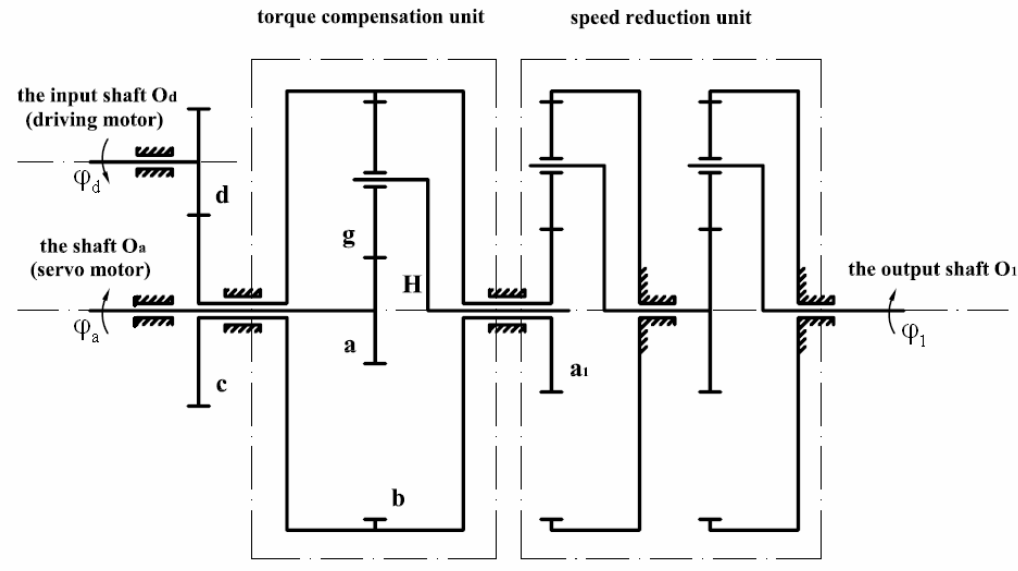

Fig.1 The internal structure of the integrated device

The differential gear train, which is a two-DOF mechanism, is composed of the sun gear $\mathbf{a}$, the planetary gear $\mathbf{g}$, the internal gear $\mathbf{b}$ and the planetary carrier $\mathbf{H}$. The sun gear $\mathbf{a}$, which is one of the two input members of the differential gear train, is driven by the servo motor through the shaft $\mathbf{O}_{\mathbf{a}}$. A changeable control function is provided by the servo motor to 
drive the sun gear a for reducing the torque fluctuation of the machine. The internal gear $\mathbf{b}$, which is the other input member of the differential gear train, is connected with the gears $\mathbf{c}$ and $\mathbf{a}_{1}$. Here, the gear $\mathbf{c}$ is in mesh with the gear $\mathbf{d}$ which is installed on the shaft $\mathbf{O}_{\mathbf{d}}$. The gear $\mathbf{a}_{1}$ is the sun gear of the first stage planetary gear train. The driving speed and torque is transmitted through the gears $\mathbf{d}, \mathbf{c}, \mathbf{b}$ and $\mathbf{a}_{1}$ to the two-stage planetary gear train and output through the shaft $\mathbf{O}_{1}$ to the working mechanism.

Figure 2 displays the three dimensional model of the integrated device. Here, the servo motor is embedded within a sleeve and fixed to the case. The gears $\mathbf{c}$ and $\mathbf{b}$ are integrated together and connected to the gear $\mathbf{a}_{1}$.

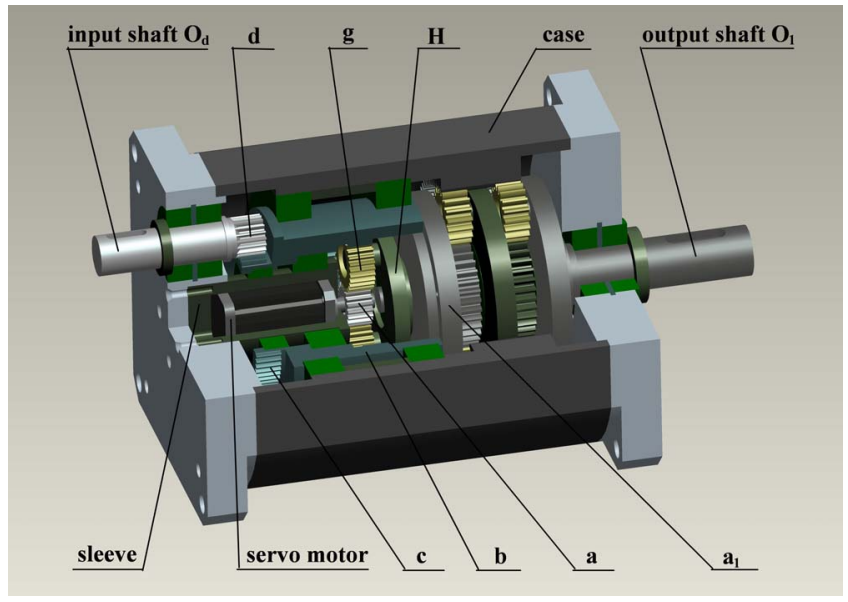

Fig.2 The three dimensional model of the integrated device

The main features of the proposed integrated device are listed as follows. First of all, this integrated device can not only transmit the driving speed and torque to the working mechanism, but also minimize the torque fluctuation of the machine. Besides, when reducing the torque fluctuation, the torque compensation unit will affect neither the work of the speed reduction unit nor the kinematics characteristics of the working mechanism. What's more, if the working condition is altered, the torque compensation unit of the proposed device can balance the torque fluctuation all the same by adjusting the speed trajectory of the servo motor. In addition, when the working mechanism is replaced by another one, as long as the requirement of the speed reduction unit is the same as the old system, the torque compensation unit can also minimize the torque fluctuation of the new system.

\section{Torque Analysis}

In this section, the balancing principle of the proposed integrated device is analyzed. As shown in Fig.1, the angular displacements of the input members $\mathbf{d}$ and $\mathbf{a}$ are represented by the symbols $\varphi_{d}$ and $\varphi_{a}$ respectively. Correspondingly, the angular velocities and the angular accelerations of them are denoted by $\dot{\varphi}_{d}, \dot{\varphi}_{a}, \ddot{\varphi}_{d}$ and $\ddot{\varphi}_{a}$ respectively. The input member of the working mechanism will be connected to the output shaft $\mathbf{O}_{1}$, and its angular displacement and angular velocity are given by $\varphi_{1}$ and $\dot{\varphi}_{1}$ respectively. Let $i_{1}$ and $i_{2}$ denote the transmission ratios of $\mathbf{d}-\mathbf{c}$ and the two-stage planetary gear train respectively. Then, the transmission ratio of the whole device is

$$
i=i_{1} i_{2} \text {. }
$$

Since the rotation direction of the gear $\mathbf{d}$ is opposite to the direction of gear $\mathbf{c}, i_{1}$ is a negative value here. There are two cases need to be compared: the integrated device without the torque compensation unit and the integrated device with the torque compensation unit. Considering the VDI guideline ${ }^{(22)}$ that the lowest natural frequency of vibration essentially exceeds the rotational frequency of the driving crank at least tenfold and vibrations do not 
have an influence, an assumption is made in the model that all bodies are rigid. Moreover, the analysis is also based on the following assumptions: the angular velocity $\dot{\varphi}_{d}$ is constant; joint clearances, friction forces and gravity forces are negligible; each moving part is homogeneous and its center of mass is on its own geometric center.

\subsection{The integrated device without the torque compensation unit}

In the case of the integrated device without the torque compensation unit, the gear $\mathbf{c}$ is connected to the sun gear $\mathbf{a}_{1}$ directly. Let $E_{1}$ and $E_{2}$ denote the kinetic energies of the working mechanism and the device without the torque compensation unit respectively. Then, the total kinetic energy of this case $E^{\prime}$ can be expressed as

$$
E^{\prime}=E_{1}+E_{2} .
$$

In which, $E_{1}$ can be formulated as

$$
E_{1}=\frac{1}{2} J_{e}\left(\varphi_{1}\right) \dot{\varphi}_{1}^{2} .
$$

$J_{e}$ is the equivalent moment of inertia of the working mechanism, and it can be written in terms of $\varphi_{1} . E_{2}$ can be derived as

$$
E_{2}=\frac{1}{2} \cdot J \cdot \dot{\varphi}_{d}^{2} .
$$

$J$ represents the equivalent moment of inertia of the device without the torque compensation unit, and it has no relationship with $\varphi_{d}$ or $\dot{\varphi}_{d}$. Then, the input torque of the driving motor $M_{d}{ }^{\prime}$ in this system can be written as

$$
M_{d}{ }^{\prime}=d E^{\prime} / d \varphi_{d}=\left(\frac{1}{2} \frac{d\left(J_{e}\left(\varphi_{1}\right)\right)}{d \varphi_{1}} \dot{\varphi}_{1}^{2}\right) \cdot \frac{1}{i} .
$$

Let

$$
M_{1}=\frac{1}{2} \frac{d\left(J_{e}\left(\varphi_{1}\right)\right)}{d \varphi_{1}} \dot{\varphi}_{1}^{2}
$$

$M_{d}{ }^{\prime}$ can be derived as

$$
M_{d}{ }^{\prime}=\frac{1}{i_{1} i_{2}} M_{1}=\frac{1}{i} M_{1} .
$$

\subsection{The integrated device with the torque compensation unit}

In the case of the integrated device with the torque compensation unit, the whole working system is a two-DOF mechanism. The motion of this system can be determined by $\varphi_{d}$ and $\varphi_{a}$. Let $E_{3}$ denote the kinetic energy of the differential gear train, the whole system's kinetic energy $E$ can be expressed as

$$
E=E_{1}+E_{2}+E_{3}
$$

where $E_{3}$ can be formulated as

$$
E_{3}=\frac{1}{2}\left(\sum J_{v} \dot{\varphi}_{v}^{2}+n J_{g} \dot{\varphi}_{g}^{2}+n J_{g g} \dot{\varphi}_{H}^{2}\right) . \quad(v=a, H, b)
$$

In Eq. (9), $J_{a}, J_{H}, J_{b}$ and $J_{g}$ are the moments of inertia with respect to the centers of mass for parts $\mathbf{a}, \mathbf{H}, \mathbf{b}$ and $\mathbf{g}$ respectively. $\dot{\varphi}_{H}, \dot{\varphi}_{b}$ and $\dot{\varphi}_{g}$ are the angular velocities of parts $\mathbf{H}, \mathbf{b}$ and g respectively. $n$ is the number of the planetary gears. It is worth noting that the planetary gear of the differential gear train revolves around not only the center of itself but also the center of the sun gear. Hence, the planetary gear has moments of inertia with respect to both its own centroid and the centroid of the sun gear. The moment of inertia with respect to the centroid of the sun gear is denoted by $J_{g g}$. The angular velocity of this revolution equals the angular velocity of the planetary carrier $\dot{\varphi}_{H}$.

Since $\dot{\varphi}_{H}$ and $\dot{\varphi}_{g}$ can be written as functions of $\dot{\varphi}_{d}$ and $\dot{\varphi}_{a}$, at the same time, $\dot{\varphi}_{b}$ and $\dot{\varphi}_{1}$ can be written as functions of $\dot{\varphi}_{d}, E$ can be derived as

$$
E=\frac{1}{2} J_{11} \dot{\varphi}_{d}^{2}+J_{12} \dot{\varphi}_{d} \dot{\varphi}_{a}+\frac{1}{2} J_{22} \dot{\varphi}_{a}^{2} .
$$


Where $J_{11}, J_{12}$ and $J_{22}$ are the equivalent moments of inertia of the whole system. They can be derived as

$$
\begin{aligned}
& J_{11}=J_{e}\left(\varphi_{1}\right)\left(1 / i^{2}\right)+J+J_{b} k_{1}^{2}+J_{H} k_{1}^{2} k_{5}^{2}+n J_{g} k_{1}^{2} k_{3}^{2}+n J_{g g} k_{1}^{2} k_{5}^{2}, \\
& J_{12}=-J_{H} k_{1} k_{4} k_{5}+n J_{g} k_{1} k_{2} k_{3}-n J_{g g} k_{1} k_{4} k_{5}, \\
& J_{22}=J_{a}+J_{H} k_{4}^{2}+n J_{g} k_{2}^{2}+n J_{g g} k_{4}^{2} .
\end{aligned}
$$

In which,

$$
\begin{aligned}
& k_{1}=z_{d} / z_{c}=-1 / i_{1}, \\
& k_{2}=z_{a} /\left(z_{b}-z_{a}\right), \\
& k_{3}=z_{b} /\left(z_{b}-z_{a}\right), \\
& k_{4}=z_{a} /\left(z_{a}+z_{b}\right), \\
& k_{5}=z_{b} /\left(z_{a}+z_{b}\right) .
\end{aligned}
$$

Here, $z_{a}, z_{b}, z_{c}$ and $z_{d}$ are the teeth numbers of the gears $\mathbf{a}, \mathbf{b}, \mathbf{c}$ and $\mathbf{d}$ respectively.

According to the Lagrange's equation of the system, the input torques of the driving motor and the servo motor $M_{d}$ and $M_{a}$ can be expressed as

$$
\begin{aligned}
& M_{d}=J_{11} \ddot{\varphi}_{d}+J_{12} \ddot{\varphi}_{a}+(1 / i) M_{1}, \\
& M_{a}=J_{12} \ddot{\varphi}_{d}+J_{22} \ddot{\varphi}_{a} .
\end{aligned}
$$

Because there is an assumption that the angular velocity $\dot{\varphi}_{d}$ is constant, then, we have $\ddot{\varphi}_{d}=0$. So Eq. (13) can be derived as

$$
\begin{aligned}
& M_{d}=J_{12} \ddot{\varphi}_{a}+(1 / i) M_{1}, \\
& M_{a}=J_{22} \ddot{\varphi}_{a} .
\end{aligned}
$$

Equation (14a) indicates that if

$$
\ddot{\varphi}_{a}=-M_{1} /\left(J_{12} \cdot i\right),
$$

the input torque of the driving motor $M_{d}$ will equal zero:

$$
M_{d}=0 \text {. }
$$

Based on the analysis above, a control function of the servo motor just like Eq. (15) that can totally eliminate the input torque of the driving motor is obtained. When $M_{d}$ is eliminated, the input torque of the servo motor $M_{a}$ can be derived as

$$
M_{a}=-\left(J_{22} / J_{12}\right) \cdot(1 / i) M_{1} .
$$

According to Eq. (11b), we have

$$
J_{12}=S \cdot k_{1}=-S / i_{1},
$$

where,

$$
S=-J_{H} k_{4} k_{5}+n J_{g} k_{2} k_{3}-n J_{g g} k_{4} k_{5} .
$$

Submitting Eq. (18) into Eq. (17) gives

$$
M_{a}=\left(J_{22} / S\right) \cdot\left(1 / i_{2}\right) M_{1} .
$$

A comparison of Eqs. (7) and (20) shows that when

$$
\left|J_{22} / S\right|<\left|1 / i_{1}\right|,
$$

the fluctuation of $M_{a}$ will be less than $M_{d}{ }^{\prime}$ 's. From Eqs. (11c) and (19) we can know that the absolute value of $J_{22} / S$ can be minimized by adjusting the structure parameters of the differential gear train. In next section, an optimization procedure is designed for limiting the input torque fluctuation of the servo motor $M_{a}$ in the situation that $M_{d}$ is eliminated completely. Besides, other two optimization methods are developed in next section for getting satisfactory tradeoffs between the fluctuations of both $M_{d}$ and $M_{a}$.

\section{Optimization Design}

Three optimization procedures are designed in this section. First of all, the structure parameters of the differential gear train are adjusted to limit the fluctuation of the input torque $M_{a}$ in the situation that $M_{d}$ is totally eliminated. Furthermore, if we want to reduce 
the total torque fluctuation of the system rather than only eliminate the fluctuation of $M_{d}$, when the structure parameters of the differential gear train are unchangeable, an optimization approach can be used to find a suitable control function of the servo motor for getting a satisfactory tradeoff between $M_{d}$ and $M_{a}$. In addition, an integrated design of the structure parameters of the differential gear train and the control function of the servo motor is developed for minimizing the total torque fluctuation of the system.

\subsection{Structure parameters design}

The optimization procedure of minimizing the absolute value of $J_{22} / S$ is designed as follows.

\subsubsection{Objective function}

The objective function is defined as

$$
F(x)=\left|J_{22} / S\right| \text {. }
$$

The aim of this optimization design is to reduce the value of $F(x)$.

\subsubsection{Design variables}

From Eqs. (11c), (12) and (19) we can know that the design variables of this procedure are

$$
X=\left[J_{a}, J_{H}, J_{g}, J_{g g}, z_{a}, z_{b}\right] .
$$

\subsubsection{Design constrains}

The analyses of the design constraints are listed as follows.

First of all, Since the moments of inertia can neither equal zero nor less than zero, $J_{a}, J_{H}$ and $J_{g}$ should be positive. Besides, they should have upper limits. Then, we have constraints:

$$
\delta_{1}<J_{\varepsilon}<\delta_{2} . \quad(\varepsilon=a, H, g) \quad\left(\delta_{1}, \delta_{2}>0\right)
$$

Here, $\delta_{1}$ and $\delta_{2}$ are two random positive values that should be decided according to the situation of practice.

Furthermore, the relationship between $J_{g}$ and $J_{g g}$ is analyzed as follows.

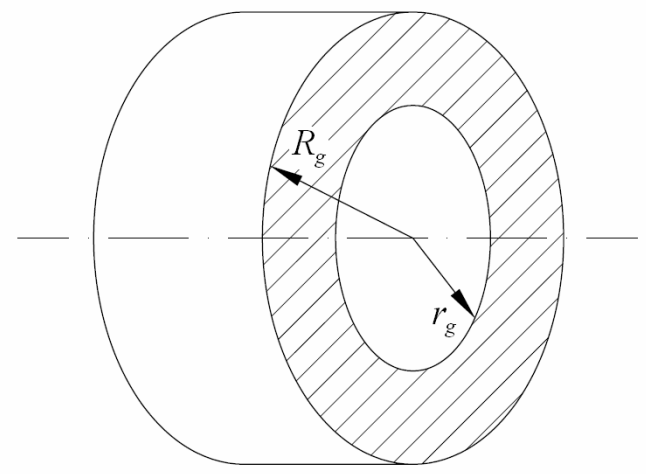

Fig.3 The scheme of the structure of the gear $\mathbf{g}$

Suppose the gear $\mathbf{g}$ is an approximate hollow circular cylinder, its scheme of structure is shown in Fig.3. $R_{g}$ and $r_{g}$ are the radiuses of the pitch circle and the hollow circle respectively. Then we have

$$
J_{g}=\frac{1}{2} m_{g}\left(R_{g}^{2}+r_{g}^{2}\right),
$$

where $m_{g}$ is the mass of the gear $\mathbf{g}$. Moreover, $J_{g g}$ can be derived as

$$
J_{g g}=J_{g}+m_{g} A^{2},
$$

where $A$ is the center distance between the gears $\mathbf{a}$ and $\mathbf{g}$, and it can be expressed as

$$
A=\left(\frac{z_{a}+z_{b}}{4}\right) M,
$$

where $M$ is the module of the gears $\mathbf{a}, \mathbf{b}$ and $\mathbf{g}$. 
According to Eq. (25), if the gear $\mathbf{g}$ is a solid cylinder, $r_{g}=0$, if it is a thin-walled cylinder, $r_{g} \approx R_{g}$. Then we obtain

$$
\frac{1}{2} m_{g} R_{g}^{2}<J_{g}<m_{g} R_{g}^{2} .
$$

Since $R_{g}$ can be formulated as

$$
R_{g}=\left(\frac{z_{b}-z_{a}}{4}\right) M
$$

the relationship between $J_{g}$ and $J_{g g}$ can be expressed as

$$
\left(1+\left(\frac{z_{a}+z_{b}}{z_{b}-z_{a}}\right)^{2}\right) J_{g}<J_{g g}<\left(1+2\left(\frac{z_{a}+z_{b}}{z_{b}-z_{a}}\right)^{2}\right) J_{g} .
$$

Additionally, suppose the gears $\mathbf{a}, \mathbf{b}$ and $\mathbf{g}$ are standard involute gears, thus the pressure angle is $\alpha=20^{\circ}$ and the addendum coefficient is $h_{a}{ }^{*}=1$. In order to keep the gears not to be undercut, the teeth numbers of them should be no less than 17 . So, at first, we have

$$
\begin{aligned}
& z_{a}>17, \\
& z_{g}>17 .
\end{aligned}
$$

Here, $z_{g}$ is the tooth number of the gear g. According to the relationship among $z_{a}, z_{b}$ and $z_{g}$ :

$$
z_{g}=\frac{1}{2}\left(z_{b}-z_{a}\right)
$$

another constraint can be gained:

$$
z_{b}>z_{a}+34 \text {. }
$$

Moreover, the teeth numbers should have upper limits too, so we have

$$
z_{b}<\delta_{3}, \quad\left(\delta_{3}>17\right)
$$

where, $\delta_{3}$ is a random positive integer that should be decided according to the situation of practice.

The design constraints of this procedure are Eqs. (24), (30), (31), (34) and (35).

This optimization problem is a nonlinear constraint optimization problem, so the fmincon function of the Matlab Optimization Toolbox can be used to solve it. This function includes a Sequential Quadratic Programming (SQP) technique and gets a local optimal solution.

\subsection{Control function design}

An optimization procedure is designed as follows for finding an appropriate control function of the servo motor.

\subsubsection{Objective function}

The objective function is defined as

$$
F(x)=\omega_{1} f_{1}(x)+\omega_{2} f_{2}(x),
$$

where

$$
\begin{aligned}
& f_{1}(x)=\sqrt{\frac{1}{\tau} \int_{0}^{\tau}\left|M_{d}\right|^{2} d t} / \sqrt{\frac{1}{\tau} \int_{0}^{\tau}\left|M_{d}\right|^{2} d t}, \\
& f_{2}(x)=\sqrt{\frac{1}{\tau} \int_{0}^{\tau}\left|M_{a}\right|^{2} d t} / \sqrt{\frac{1}{\tau} \int_{0}^{\tau}\left|M_{d}\right|^{2} d t}
\end{aligned}
$$

are used to minimize the root-mean-square (RMS) values of the input torques $M_{d}$ and $M_{a}$. $\tau$ is the period of a complete motion cycle. $\omega_{1}$ and $\omega_{2}$ are weighting factors which can be adjusted to meet various design requirements. Here, we define $\omega_{1}+\omega_{2}=1 \quad\left(\omega_{1}, \omega_{2} \geq 0\right)$. When there is no torque compensation unit in the working system, we define $\omega_{1}=1, \omega_{2}=0$. And under this situation, $F(x)=1$. The aim of this optimization design is to minimize the value of $F(x)$. A trial-and-error procedure is needed here to find the appropriate weighting 
factors.

\subsubsection{Design constraints}

In what follows, design constraints are developed for this procedure.

The sun gear a which is driven by the servo motor should rotate one revolution in a period $\tau$. Therefore, we have

$$
\varphi_{a}(\tau)-\varphi_{a}(0)=2 \pi
$$

Considering the continuity of the input motion, the angular displacement function should be at least second-order differentiable. This continuity condition can be formulated by

$$
\begin{aligned}
& \dot{\varphi}_{a}(\tau)=\dot{\varphi}_{a}(0), \\
& \ddot{\varphi}_{a}(\tau)=\ddot{\varphi}_{a}(0) .
\end{aligned}
$$

\subsubsection{Design variables}

The input trajectory $\varphi_{a}$ can be expressed as a polynomial function like

$$
\varphi_{a}(t)=\sum_{\eta=1}^{10} C_{\eta} t^{\eta}
$$

Taking the first derivative and the second derivative with respect to time $t$ respectively leads to

$$
\begin{array}{ll}
\dot{\varphi}_{a}(t)=\sum_{\eta=1}^{10} \eta C_{\eta} t^{\eta-1}, & t \in[0, \tau] \\
\ddot{\varphi}_{a}(t)=\sum_{\eta=1}^{10} \eta(\eta-1) C_{\eta} t^{\eta-2} . & t \in[0, \tau]
\end{array}
$$

Where the coefficients $C_{0}, C_{1}, C_{2}, \ldots, C_{10}$ are determined by the optimization approach for selecting a suitable control function of the servo motor. According to Eq. (37a), $C_{0}$ can be eliminated. So the design variables can be expressed as

$$
X=\left[C_{1}, C_{2}, \ldots, C_{10}\right] .
$$

Since the equality constraints can be eliminated by an algebraic method, this optimization problem can be solved by using the fminsearch function of the Matlab Optimization Toolbox. This function applies Simplex algorithm and also gets struck in local optima.

\subsection{Integrated design of structure parameters and control function}

Usually, the optimization design of the structural parameter and the control function are carried out in sequence. It is seldom to design them at the same time. Sometimes, the result gained from designing these two stages orderly may not be the best result of the entire system but just a feasible one.

The method of integrated design studies how to design the structural parameters and the control function at the same time. The objective of this optimization procedure is to find a result that includes a set of suitable structural parameters and an appropriate control function for the whole system.

\subsubsection{Objective function}

The objective function is the same as Eq. (36).

\subsubsection{Design constraints}

The design constraints are similar with Eqs. (24), (30), (31), (34), (35) and (37).

\subsubsection{Design variables}

Adding $J_{a}, J_{H}, J_{g}, J_{g g}, z_{a}$ and $z_{b}$ to this procedure, the design variables can be written as

$$
X=\left[C_{1}, C_{2}, \ldots, C_{10}, J_{a}, J_{H}, J_{g}, J_{g g}, z_{a}, z_{b}\right] .
$$

This procedure is a nonlinear constraint optimization problem, so the fmincon function of the Matlab Optimization Toolbox can be used to solve it. 


\section{Examples}

In this section, different torque compensation units of an integrated device are designed to meet different requirements. In example 1, a slider-crank mechanism is used as the working mechanism. It consists of a crank, a link and a slider. According to the optimization procedures in section 4 , three different torque compensation units of the integrated device are designed for this working mechanism. In example 2, the working mechanism is replaced by the other slider-crank mechanism, but the required transmission ratio is the same to the example 1 . The integrated device designed for the example 1 can still be used in this changed system for transmitting the driving speed and torque and balancing the torque fluctuation by redesigning a control function of the servo motor.

The structure parameters of the working mechanisms used in the examples are listed in Table 1.The required angular velocity of the crank is $150 \mathrm{rpm}$. The angular speed of the driving motor is $3000 \mathrm{rpm}$. Since the rotation direction of the output shaft $\mathbf{O}_{\mathbf{1}}$ is opposite to the rotation direction of the input shaft $\mathbf{O}_{\mathbf{d}}$, the transmission ratio of the whole integrated device is $i=-20$. Let $i_{1}=-4$ and $i_{2}=5$. In order to provide better balancing of the gear tooth loads and inertia force, two planetary gears are employed in the differential gear train and the two-stage planetary gear train respectively, so we have $n=2$.

Table 1 Parameters of the slider-crank mechanisms used in the examples

\begin{tabular}{|c|c|c|c|c|c|c|}
\hline & \multicolumn{3}{|c|}{ Slider-crank mechanism 1 } & \multicolumn{3}{c|}{ Slider-crank mechanism 2 } \\
\hline & Crank & Link & Slider & Crank & Link & Slider \\
\hline Length (m) & 0.1 & 0.4 & - & 0.12 & 0.3 & - \\
\hline Mass (kg) & 0.05 & 0.2 & 1 & 0.08 & 0.15 & 1.3 \\
\hline
\end{tabular}

\subsection{Example 1}

In this example, the slider-crank mechanism 1 shown in Table 1 is used as the working mechanism. Firstly, the structure parameters of the differential gear train are adjusted to limit the fluctuation of the input torque $M_{a}$ in the situation that $M_{d}$ is totally eliminated. Secondly, a satisfactory tradeoff between $M_{d}$ and $M_{a}$ is obtained by optimizing the control function of the servo motor. Thirdly, the integrated design of the structure parameters of the differential gear train and the control function of the servo motor is implemented for minimizing the fluctuations of both $M_{d}$ and $M_{a}$.

\subsubsection{Example 1.1}

According to Eq. (15), a control function of the servo motor which can completely eliminate $M_{d}$ is gained for the slider-crank mechanism 1. It is shown in Fig.4 (a). In this example, the fluctuation of $M_{a}$ is limited through adjusting the structure parameters of the differential gear train. Let $\delta_{1}=1 \times 10^{-6} \mathrm{~kg} \cdot \mathrm{m}^{2}, \delta_{2}=1 \mathrm{~kg} \cdot \mathrm{m}^{2}$ and $\delta_{3}=150$. The results of this optimization procedure are shown in Fig. 4 and listed in Tables 3 and 4.

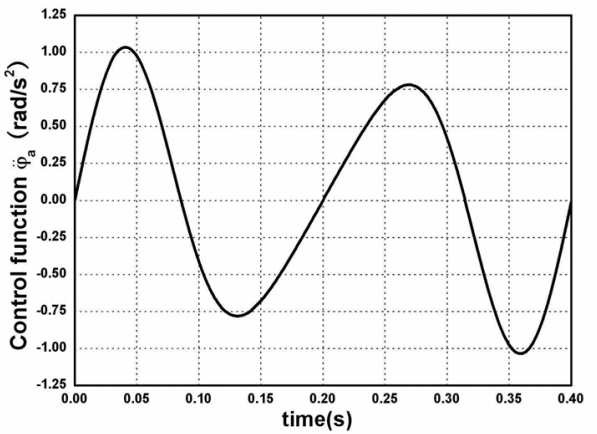

(a) Control function $\ddot{\varphi}_{a}$

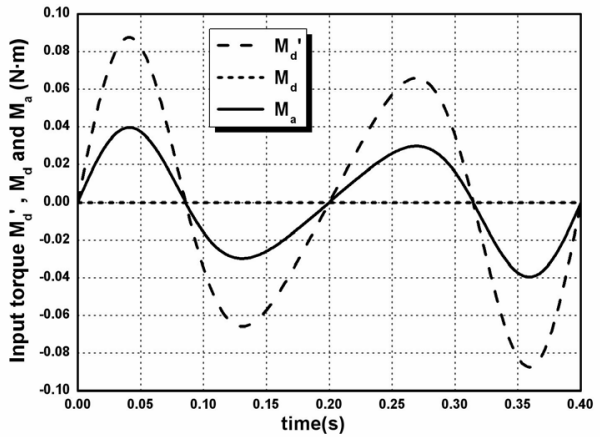

(b) Input torque $M_{d}{ }^{\prime}, M_{d}$ and $M_{a}$

Fig.4 Design results of example 1.1

From Fig.4 (b) we can see that the fluctuation of $M_{a}$ is limited by optimizing the structure parameters of the differential gear train. As shown in Table 3, an improvement of $54.64 \%$ is obtained by comparing the value of $F(x)$ with $\left|1 / i_{1}\right|=0.25$. 


\subsubsection{Example 1.2}

The control function of the servo motor is adjusted in this example for balancing the input torque fluctuations of both $M_{d}$ and $M_{a}$. The structure parameters of the differential gear train used in this example are listed in Table 2. According to Eq. (37), three of the design variables which listed in Eq. (39) can be eliminated. Here, we eliminate $C_{8}, C_{9}$ and $C_{10}$. After a trail-and-error procedure, $\omega_{1}=0.62$ and $\omega_{2}=0.38$ are chosen as the weighting factors in this example. The results are shown in Fig.5 and listed in Tables 3 and 4.

Table 2 Parameters of the differential gear train used in examples 1.2, 2.1 and 2.2

\begin{tabular}{|c|c|c|}
\hline & Tooth number & Moment of Inertia $\left(\mathrm{kg} \cdot \mathrm{m}^{2}\right)$ \\
\hline Sun gear a & 24 & $J_{a}=2 \times 10^{-4}$ \\
\hline \multirow{2}{*}{ Planetary gear $\mathbf{g}$} & \multirow{2}{*}{36} & $J_{g}=3 \times 10^{-4}$ \\
\cline { 2 - 3 } & & $J_{g g}=1.5 \times 10^{-3}$ \\
\hline Internal gear b & 96 & - \\
\hline Planetary carrier $\mathbf{H}$ & - & $J_{H}=0.8$ \\
\hline
\end{tabular}

According to the data listed in Table 3, the improvements of $40.07 \%, 34.06 \%$ and $38.40 \%$ for the RMS value, the maximal value and the minimal value of $M_{d}$ respectively are gained by comparing with the values of $M_{d}{ }^{\prime}$. Similarly, the improvements of $43.63 \%$, $56.46 \%$ and $17.26 \%$ for the RMS value, the maximal value and the minimal value of $M_{a}$ respectively are gained. The reduction of $F(x)$ is $41.42 \%$.

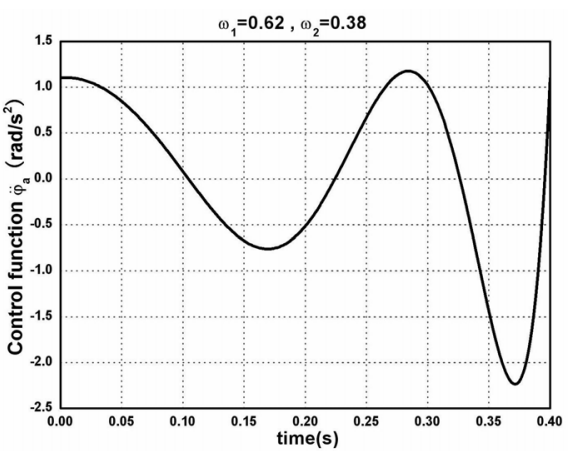

(a) Control function $\ddot{\varphi}_{a}$

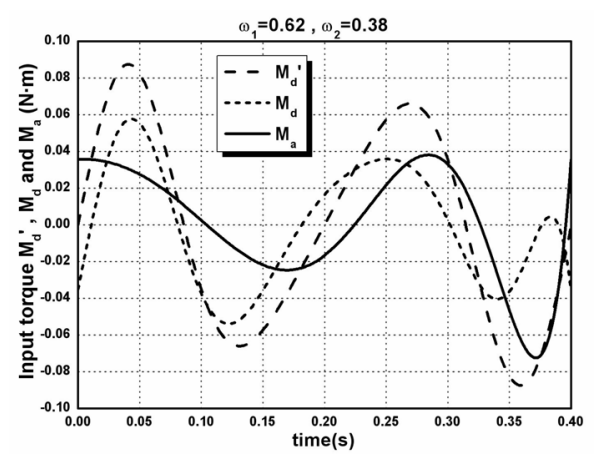

(b) Input torque $M_{d}{ }^{\prime}, M_{d}$ and $M_{a}$

Fig.5 Design results of example 1.2

\subsubsection{Example 1.3}

The integrated design of the structure parameters of the differential gear train and the control function of the servo motor is taken in this example. Here, we also let $\delta_{1}=1 \times 10^{-6} \mathrm{~kg} \cdot \mathrm{m}^{2}, \delta_{2}=1 \mathrm{~kg} \cdot \mathrm{m}^{2}$ and $\delta_{3}=150$. The design variables $C_{8}, C_{9}$ and $C_{10}$ are eliminated too. The weighting factors $\omega_{1}=0.9$ and $\omega_{2}=0.1$ are selected in this example. The results are shown in Fig.6 and listed in Tables 3 and 4.

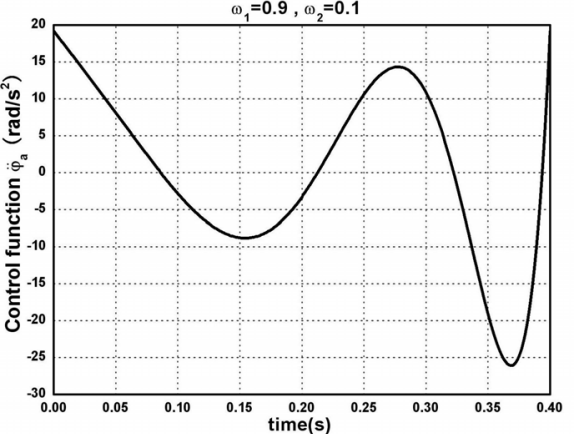

(a) Control function $\ddot{\varphi}_{a}$

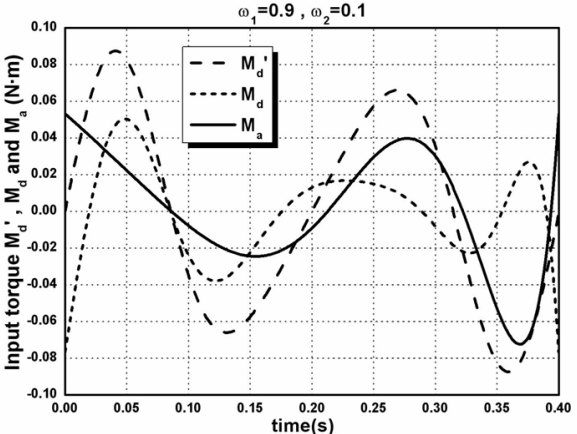

(b) Input torque $M_{d}{ }^{\prime}, M_{d}$ and $M_{a}$

Fig.6 Design results of example 1.3 
From the results listed in Table 3 we can find that the improvements of the RMS value, the maximal value and the minimal value of $M_{d}$ are $53.31 \%, 42.51 \%$ and $11.77 \%$ respectively. At the same time, the improvements for the RMS value, the maximal value and the minimal value of $M_{a}$ are $40.98 \%, 39.2 \%$ and $17.26 \%$ respectively. The value of $F(x)$ is decreased by $52.07 \%$. Comparing with example 1.2 , the reduction of $F(x)$ in this example is $10.65 \%$ more than that of example 1.2. Moreover, from the design variables of examples 1.1 and 1.3 which listed in Table 4 we can find that $J_{a}, J_{g}$ and $z_{a}$ are equal to the boundaries of the optimization constrains respectively. So we get a conclusion that the optimization results will be better with smaller values of $J_{a}, J_{g}$ and $z_{a}$.

\subsection{Example 2}

In this example, the slider-crank mechanism 2 shown in Table 1 is used as the working mechanism. The structure parameters of the differential gear train listed in Table 2 are applied here too. At first, the control function of the servo motor gained from example 1.2 is still used for minimizing the input torque fluctuations of the changed system. What's more, an optimization approach is applied to redesign a control function of the servo motor for the new system.

\subsubsection{Example 2.1}

With the control function obtained from example 1.2, the input torque fluctuations of the changed system can also be reduced. The results are shown in Fig.7 and listed in Tables 3 and 4 .

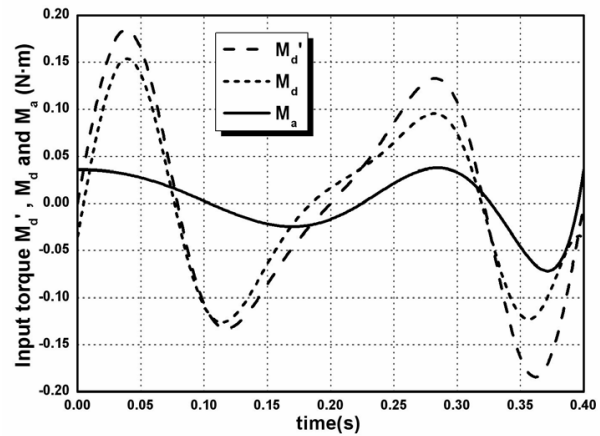

Fig.7 Input torque $M_{d}{ }^{\prime}, M_{d}$ and $M_{a}$ of example 2.1

After balanced by the control function which is obtained from example 1.2, the new system has some improvements for the RMS value, the maximal value and the minimal value of $M_{d}$. These improvements are $22.64 \%, 16.65 \%$ and $31.34 \%$ respectively. The RMS value, the maximal value and the minimal value of $M_{a}$ also have some improvements. They are $71.88 \%, 79.50 \%$ and $61.12 \%$ respectively. These data indicate that slight improvements of $M_{d}$ are gained by using the control function of example 1.2 .

\subsubsection{Example 2.2}

In this example, a control function is redesigned for the changed system. The design variables $C_{8}, C_{9}$ and $C_{10}$ can also be eliminated. With a trail-and-error procedure, $\omega_{1}=0.62$ and $\omega_{2}=0.38$ are selected as the appropriate weighting factors too. The results are shown in Fig. 8 and listed in Tables 3 and 4.

According to the data listed in Table 3, the improvements for the RMS value, the maximal value and the minimal value of $M_{d}$ are $42.82 \%, 26.08 \%$ and $25.66 \%$ respectively. Comparing with example 2.1, the improvements of $M_{d}$ in this example are $20.18 \%, 9.43 \%$ and $14.32 \%$ more than that of example 2.1 respectively. The improvements for the RMS value, the maximal value and the minimal value of $M_{a}$ are $45.27 \%, 46.20 \%$ and $30.15 \%$ respectively. The reduction of $F(x)$ is $43.75 \%$. These analytical results indicate that the balancing effect of redesigning a control function for the changed system is better than the effect of using the old control function. Hence, when the working mechanism is changed, it is necessary to redesign a new control function for the changed system. 
It is observed from Example 2 that the torque compensation unit of the proposed integrated device can balance the input torque of the changed system all the same by redesigning a control function for the servo motor.

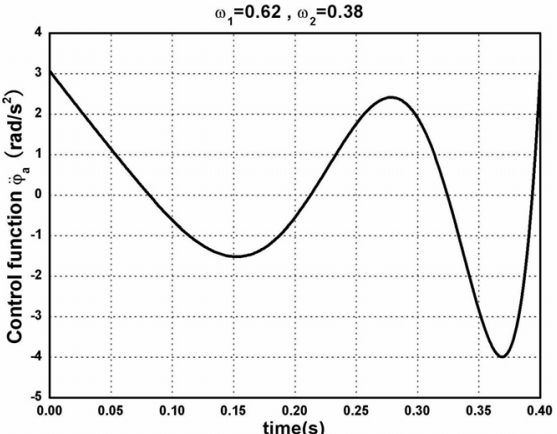

(a) Control function $\ddot{\varphi}_{a}$

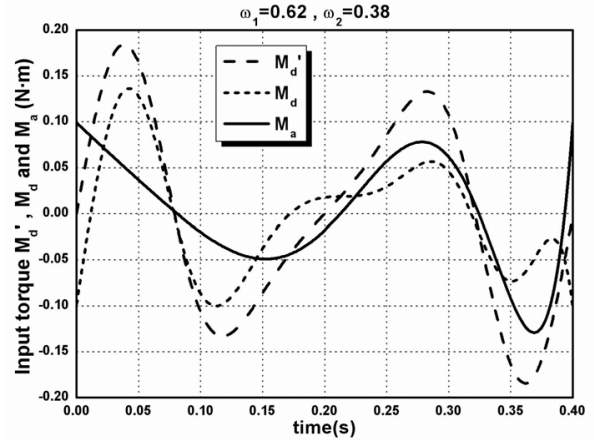

(b) Input torque $M_{d}{ }^{\prime}, M_{d}$ and $M_{a}$

Fig.8 Design results of example 2.2

Table 3 Data of the results for the examples

\begin{tabular}{|c|c|c|c|c|c|}
\hline & Example 1.1 & Example 1.2 & Example 1.3 & Example 2.1 & Example 2.2 \\
\hline$F(x)$ & 0.1134 & 0.5858 & 0.4793 & - & 0.5625 \\
\hline$f_{1}(x)$ & - & 0.5993 & 0.4669 & 0.7736 & 0.5718 \\
\hline$f_{2}(x)$ & - & 0.5637 & 0.5902 & 0.2812 & 0.5473 \\
\hline$M_{d{ }^{\prime}{ }_{\text {max }}}(\mathrm{N} \cdot \mathrm{m})$ & 0.0875 & 0.0875 & 0.0875 & 0.1844 & 0.1844 \\
\hline$M_{d{ }^{\prime}{ }_{\min }}(\mathrm{N} \cdot \mathrm{m})$ & -0.0875 & -0.0875 & -0.0875 & -0.1844 & -0.1844 \\
\hline$M_{d \max }(\mathrm{N} \cdot \mathrm{m})$ & 0 & 0.0577 & 0.0503 & 0.1537 & 0.1363 \\
\hline$M_{d \min }(\mathrm{N} \cdot \mathrm{m})$ & 0 & -0.0539 & -0.0772 & -0.1266 & -0.1002 \\
\hline$M_{a \max }(\mathrm{N} \cdot \mathrm{m})$ & 0.0397 & 0.0381 & 0.0532 & 0.0378 & 0.0992 \\
\hline$M_{a \min }(\mathrm{N} \cdot \mathrm{m})$ & -0.0397 & -0.0724 & 0.0724 & -0.0717 & -0.1288 \\
\hline
\end{tabular}

Table 4 The results of the design variables for the examples

\begin{tabular}{|c|c|}
\hline Example 1.1 & $X=\left[1 \times 10^{-6}, 0.49981,1 \times 10^{-6}, 3.3689 \times 10^{-6}, 17,150\right]$ \\
\hline Example 1.2 & $X=[15.659,0.55152,0.16106,-10.19,1.3663,-14.361,12.392]$ \\
\hline \multirow{2}{*}{ Example 1.3 } & $X=[15.177,9.5867,-35.822,-12.366,3.5541,-13.58,12.509$, \\
& $\left.1 \times 10^{-6}, 0.12666,1 \times 10^{-6}, 4.5656 \times 10^{-6}, 17,97.866\right]$ \\
\hline Example 2.1 & $X=[15.659,0.55152,0.16106,-10.19,1.3663,-14.361,12.392]$ \\
\hline Example 2.2 & $X=[15.637,1.5319,-6.5584,1.3827,-0.92262,-19.341,16.984]$ \\
\hline
\end{tabular}

\section{Conclusion}

From the examples in section 5 we can see that through selecting a suitable control function for the servo motor, the torque compensation unit of the integrated device can eliminate the input torque fluctuation of the driving motor completely. At the same time, by optimizing the structure parameters of the differential gear train, the input torque fluctuation of the servo motor can also be limited. Besides, satisfactory tradeoff between $M_{d}$ and $M_{a}$ is gained by adjusting the control function of the servo motor in the situation that the structure parameters of the differential gear train are unchangeable. What's more, a set of suitable structure parameters of the differential gear train and an appreciate control function of the servo motor are obtained by applying an integrated design procedure. Additionally, when the working mechanism is changed, the control function of the servo motor should be redesigned to minimize the torque fluctuations of the new system.

The proposed integrated device can not only transmit the speed and torque to the working mechanism, but also balance the input torque fluctuation of the machine. Since the control function of the servo motor can be adjusted to meet different requirements, the 
proposed integrated device can be used in different systems for reducing the torque fluctuations and transmitting the speed and torque universally. When the working condition is changed, the torque compensation unit of the integrated device can also balance the torque fluctuation by finding a suitable control function for the servo motor. Moreover, when minimizing the input torque fluctuation of the system, the integrated device will not disturb the desired behavior of the working mechanism.

\section{Acknowledgments}

This research is sponsored by Program for New Century Excellent Talents in University (NCET-07-0063) and National Natural Science Foundation of China (No.50405004 and No.50875018).

\section{References}

(1) Kochev, I. S., General Method for Active Balancing of Combined Shaking Moment and Torque Fluctuations in Planar Linkages, Mech. Mach. Theory, Vol.25, No.6 (1990), pp.679-687.

(2) Liu, D. Y. and Huang, Z., The Input Torque Balancing of Linkage, Mech. Mach. Theory, Vol.24, No.2 (1989), pp.99-103.

(3) Demeulenaere, B., Spaepen, P. and De Schutter, J., Input Torque Balancing Using a Cam-Based Centrifugal Pendulum: Design Procedure and Example, Journal of Sound and Vibration, Vol.283, No.1-2 (2005), pp.1-20.

(4) Demeulenaere, B., Spaepen, P. and De Schutter, J., Input Torque Balancing Using a Cam-Based Centrifugal Pendulum: Design Optimization and Robustness, Journal of Sound and Vibration, Vol.283, No.1-2 (2005), pp.21-46.

(5) Demeulenaere, B., Spaepen, P., Masselis, S. et al, Experimental Validation of Input Torque Balancing Applied to Weaving Machinery, Transaction of the ASME, Journal of Mechanical Design, Vol.130, No.2 (2008), pp.022307(1-10).

(6) Demeulenaere, B. and De Schutter, J., Input Torque Balancing Using an Inverted Cam Mechanism, Transaction of the ASME, Journal of Mechanical Design, Vol.127, No.6 (2005), pp.887-900.

(7) Lee, T. M., Lee, D. Y., Lee, H. C. and Yang, M. Y., Design of Cam Type Transfer Unit Assisted with Conjugate Cam and Torque Control Cam, Mech. Mach. Theory, Vol.44, No.6 (2009), pp.1144-1155.

(8) Nishioka, M., State of the Art of Torque Compensation of Dynamic Effects, Proceedings of the 6th World Congress on Mech. Mach. Theory, (1996), pp.713-717.

(9) Nishioka, M., Direct Type Torque Compensation Cam for Indexing Drive, Proceedings of the 11th World Congress in Mechanism and Machine Science, (2004), pp.1035-1039.

(10) Wu, C. J. and Angeles, J., The Optimum Synthesis of an Elastic Torque-Compensating Cam Mechanism, Mech. Mach. Theory, Vol.36, No.2 (2001), pp.245-259.

(11) Tsay, D. M., Ho, H. C. and Wang, K. C., Design of Torque Balancing Cams for Globoidal Cam Indexing Mechanisms, Transaction of the ASME, Journal of Mechanical Design, Vol.124, No.33 (2002), pp.441-447.

(12) Dooner, D. B., Use of Noncircular Gears to Reduce Torque and Speed Fluctuations in Rotating Shafts, Transaction of the ASME, Journal of Mechanical Design, Vol.119, No.2 (1997), pp.299-306.

(13) Yao, Y. A. and Yan, H. S., A New Method for Torque Balancing of Planar Linkages Using Non-Circular Gears, Proceedings of the Institution of Mechanical Engineers, Part C: Journal of Mechanical Engineering Science, Vol.217, No.5 (2003), pp.495-503.

(14) Funk, W. and Han, J. Y., On the Complete Balancing of the Inertia-Caused Input Torque for Plane Mechanism, ASME Design Engineering Technical Conference and Computers in Engineering Conference, Irvine, California, 18-22 August 1996, 
96-DETC/MECH-1570, (1996), pp.1-6.

(15) Teng, G. L., Fu, H. B. and Zhou, W. Y., A New Method of Torque Compensation for High Speed Indexing Cam Mechanism, Transaction of the ASME, Journal of Mechanical Design, Vol.121, No.2 (1999), pp.319-323.

(16) Thuemmel, T., Dynamic Balancing of Linkage by Active Control with Redundant Drive, Proceedings of the 9th IFTOMM World Congress, Milan, Italy, Vol.2, (1995), pp.970-975.

(17) Thuemmel, T. and Brandl, M., Active Balancing of Joint Forces in High-Speed Linkages by Redundant Drives and Learning Control, Proceedings of the 1996 ASME Design Engineering Technical Conference and Computers in Engineering Conference, Irvine, California, (1996).

(18) Dyer, S. W. and Ni, J., Adaptive Influence Coefficient Control of Single-Plane Active Balancing Systems for Rotating Machinery, Transaction of the ASME, Journal of Manufacturing Science and Engineering, Vol.123, No.2 (2001), pp.291-298.

(19) Yao, Y. A., Yan, H. S. and Zou, H. J., Dynamic Design of Variable Speed Planar Linkages, Chinese Journal of Mechanical Engineering, Vol.18, No.1 (2005), pp.51-54.

(20) Zhang, Y., Yao, Y. A. and Cha, J. Z., An Independent Active Balancer for Planar Mechanisms, Transactions of Canadian Society for Mechanical Engineering, Vol.30, No.2 (2007), pp.167-190.

(21) Sun, J. and Yao, Y. A, An Independent Active Torque Balancer Using a Servo-Controlled Differential Gear Train, Transactions of Canadian Society for Mechanical Engineering, Vol.32, No.2 (2009), pp.169-188.

(22) VDI2149, Blatt 1, Getriebedynamik-Starrkörper Mechanismen (Dynamics of Mechanisms-Rigid Body Mechanisms), Verein Deutscher Ingenieure, Richtlinien (1999). 